\title{
Controlling DNA Capture and Propagation through Artificial Nanopores
}

2007

Vol. 7, No. 9

2824-2830

\author{
Eliane H. Trepagnier, ${ }^{\dagger, \neq, \S, \|}$ Aleksandra Radenovic, ${ }^{\dagger, \S, \|}$ David Sivak,,$\neq \perp$ \\ Phillip Geissler,,,$+ \perp$ and Jan Liphardt ${ }^{\star, \neq, \S,, l}$ \\ Biophysics Graduate Group, University of California, Berkeley, Berkeley, California, \\ Department of Physics, University of California, Berkeley, Berkeley, California, \\ Physical Biosciences Division, Lawrence Berkeley National Laboratory (LBL), \\ Berkeley, California, and College of Chemistry, University of California, Berkeley, \\ Berkeley, California
}

Received June 15, 2007; Revised Manuscript Received July 30, 2007

\begin{abstract}
Electrophorescing biopolymers across nanopores modulates the ionic current through the pore, revealing the polymer's diameter, length, and conformation. The rapidity of polymer translocation $(\sim 30000 \mathrm{bp} / \mathrm{ms})$ in this geometry greatly limits the information that can be obtained for each base. Here we show that the translocation speed of $\lambda$-DNA through artificial nanopores can be reduced using optical tweezers. DNAs coupled to optically trapped beads were presented to nanopores. DNAs initially placed up to several micrometers from the pore could be captured. Subsequently, the optical tweezers reduced translocation speeds to $150 \mathrm{bp} / \mathrm{ms}$, about 200 -fold slower than free DNA. Moreover, the optical tweezers allowed us to "floss" single polymers back and forth through the pore. The combination of controlled sample presentation, greatly slowed translocation speeds, and repeated electrophoresis of single DNAs removes several barriers to using artificial nanopores for sequencing, haplotyping, and characterization of protein-DNA interactions.
\end{abstract}

The characterization of single biopolymers is one of the potential applications of artificial nanopores ${ }^{1,2}$ and nanochannels. $^{3-5}$ For example, artificial nanopores have been proposed as tools for rapid DNA sequencing. ${ }^{1,2,6-10}$ This approach is based on the modulation of the ionic current through the pore as a polymer traverses it, revealing the polymer's diameter, length, and conformation. ${ }^{1,2,6-10} \mathrm{~A}$ roadblock to single base resolution in such nanopore-based DNA sequencing approaches is a lack of control over translocation speeds. Speeds can be reduced by lowering the ionic strength or the driving voltage, ${ }^{11}$ but this comes at the cost of decreased ionic current, reducing the signal-to-noise ratio.

The widespread use of optical tweezers in single-molecule biophysics suggests their utility to control sample presentation to a pore or channel, reduce polymer propagation speeds without impairing ionic currents, and to repeatedly characterize one DNA molecule. The integration of optical trapping with synthetic nanopore translocation experiments was first

* To whom correspondence should be addressed. E-mail: Liphardt@ physics.berkeley.edu.

These authors contributed equally to this work.

$\doteqdot$ Biophysics Graduate Group, University of California, Berkeley.

$\S$ Department of Physics, University of California, Berkeley.

"Physical Biosciences Division, LBL.

${ }^{\perp}$ College of Chemistry, University of California, Berkeley. demonstrated by Keyser et al. ${ }^{12}$ These authors used optical force measurement to calculate the electrical force exerted on a DNA molecule in a nanopore as well as the effective charge of a DNA molecule in a nanopore. Here we extend their results by showing that this geometry can be used to reduce translocation rates by several hundred-fold and also repeatedly translocate and retract a single DNA.

Optical tweezers are routinely used to study mechanical properties of biopolymers (for a review, see ref 13; the details of our optical tweezers instrument are described in ref 14 and in the Supporting Information). Typically, a molecule is connected by complementary chemistry to a micrometer-scale bead that can be manipulated in three dimensions by one or more highly focused laser beams. In our experiment, $\lambda$-DNAs were connected to $10 \mu \mathrm{m}$ polystyrene beads via streptavidin-biotin linkage. As shown in the chamber schematic (Figure 1A), the trapped beads were brought into proximity of a single artificial nanopore in a membrane separating two chambers. Electrodes maintained an electrical potential across the nanopore. Nanopores were coated via atomic layer deposition (ALD) with $2-15 \mathrm{~nm}$ of alumina, ${ }^{15}$ a thermally and chemically stable insulating dielectric material. At our $\mathrm{pH}$ values $(\mathrm{pH}=7.0-8.0)$ and 


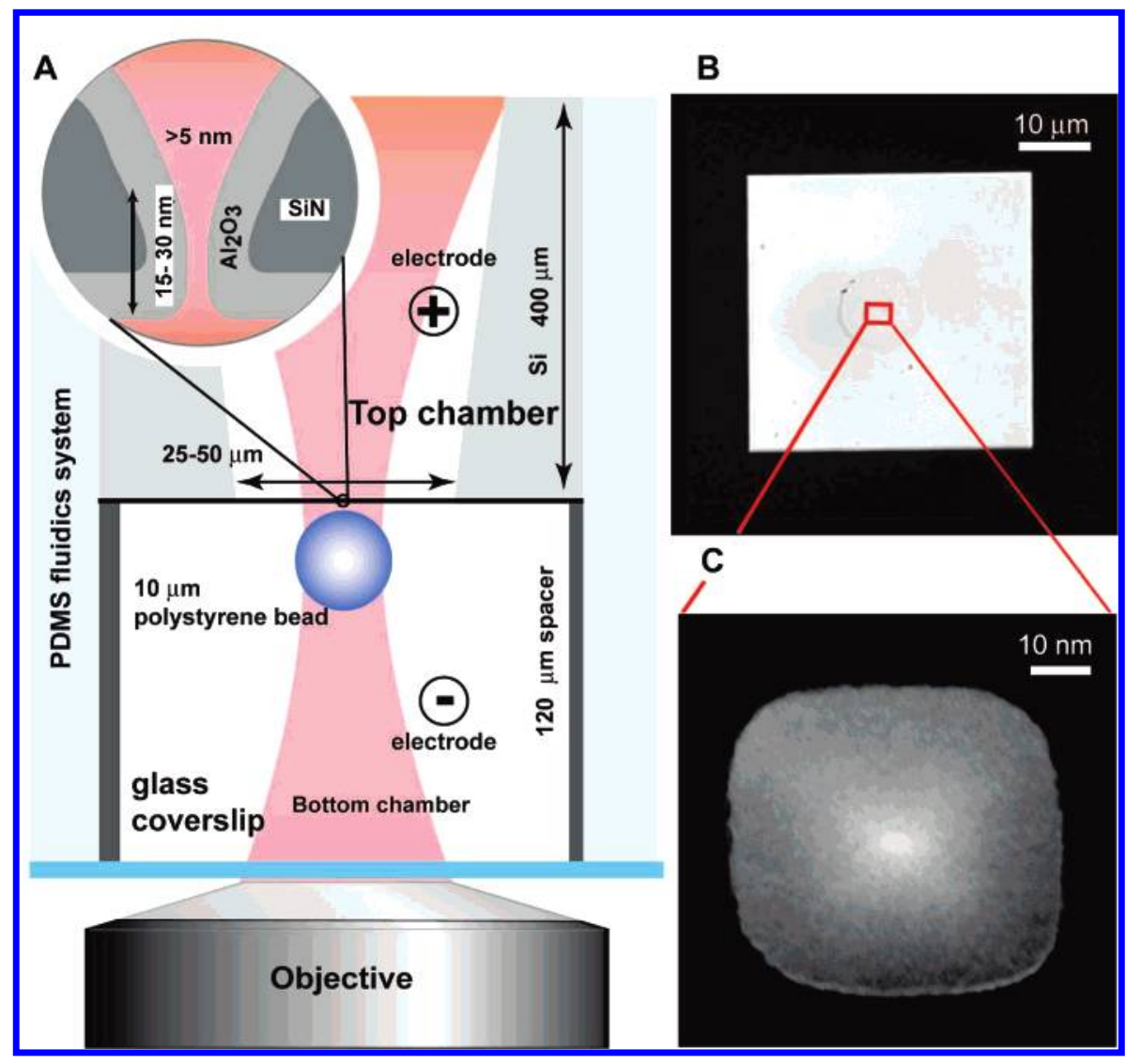

Figure 1. Schematic of experimental setup. (A) Low stress silicon nitride (LSSN) membrane containing a single nanopore divides two chambers. Electrodes are used to create and maintain an electrical potential across the nanopore. An optical trap (red) exerts mechanical forces on polymers as they translocate the pore. The nanopore membrane is supported on a $\mathrm{Si}(6 \mathrm{~mm} \times 12 \mathrm{~mm})$ chip. The silicon is etched away in a $25-50 \mu \mathrm{m}$ window of exposed LSSN. Inset: The pore is oriented so that its flat side faces the trapped bead. (B) Optical top-view image of a $50 \mu \mathrm{m}$ LSSN membrane window. (C) TEM image of a typical nanopore with a diameter of $5 \mathrm{~nm}$. To sculpt the pore to desired size, we have used a combination of ion beam sculpting and atomic layer deposition.

ionic strengths $(50 \mathrm{mM})$, alumina is positively charged and allowed excellent wetting of our coated pores.

The optical trap allows us to exert mechanical forces on polymers, as they are driven through the pore by the electrical potential. The trap also allows us to monitor the progression of the polymer through the pore via the deflection of the trapping beam, as the bead is displaced from the trap center. The measured trap stiffness was $\sim 1.1 \times 10^{-3} \mathrm{pN} / \mathrm{nm}$ per $\mathrm{mW}$ of laser power in the specimen plane. We report changes in laser deflection rather than changes in trapping force because forces are difficult to calibrate in our system. First, significant clipping of the exiting laser light is introduced by the silicon surrounding the etched membrane window. Second, our experiments occur within a few micrometers of the nanopore membrane surface, where hydrodynamic coupling influences the motions of the bead. Last, during our capture experiments, the bead travels up to $4 \mu \mathrm{m}$ from the center of the optical trap, outside of its harmonic region and thus out of range of our force measurement capabilities.

Initially, a DNA-coated bead brought into proximity of the pore experiences no net force and fluctuates about the trap minimum (Figure 2A-1). At $t=0 \mathrm{~s}$, a positive potential is applied (Figure 2B). The bead is not immediately influenced by the applied electric potential, indicating that our two force transducers, the optical trap and the electrical force, are independent. After a few seconds (typically 2$30 \mathrm{~s}$ ), the bead suddenly moves toward the pore membrane due to the capture and electric-field-driven translocation of DNA through the pore (Figure 2A-2). Ultimately, the electrical force on the DNA overpowers the optical trap, pulling the bead out of the trap (Figure 2A-3). Once the potential is reversed, the polymer is retracted though the pore and the bead relaxes into the optical trap center; the laser deflection signal consequently returns to its original value (Figure 2A-4).

Four observations convinced us that bead displacements are due to DNA threading. First, streptavidin-coated beads not incubated with $\lambda$-DNA produced no threading signatures. Second, the threading behavior correlated with applied voltage: threading only occurred when the applied voltage was positive and the threading signals ceased once the potential was reversed (Figure 2A-4). Third, some beads incubated with DNA did not exhibit threading, probably because they lacked attached DNA on the pore-proximal side of the bead. Finally, for a given bead, its threading behavior was repeatable: a bead exhibited either reproducible threading or lack of threading.

We first investigated the dynamics of DNA capture by the pore. We measured the time required for DNA capture 


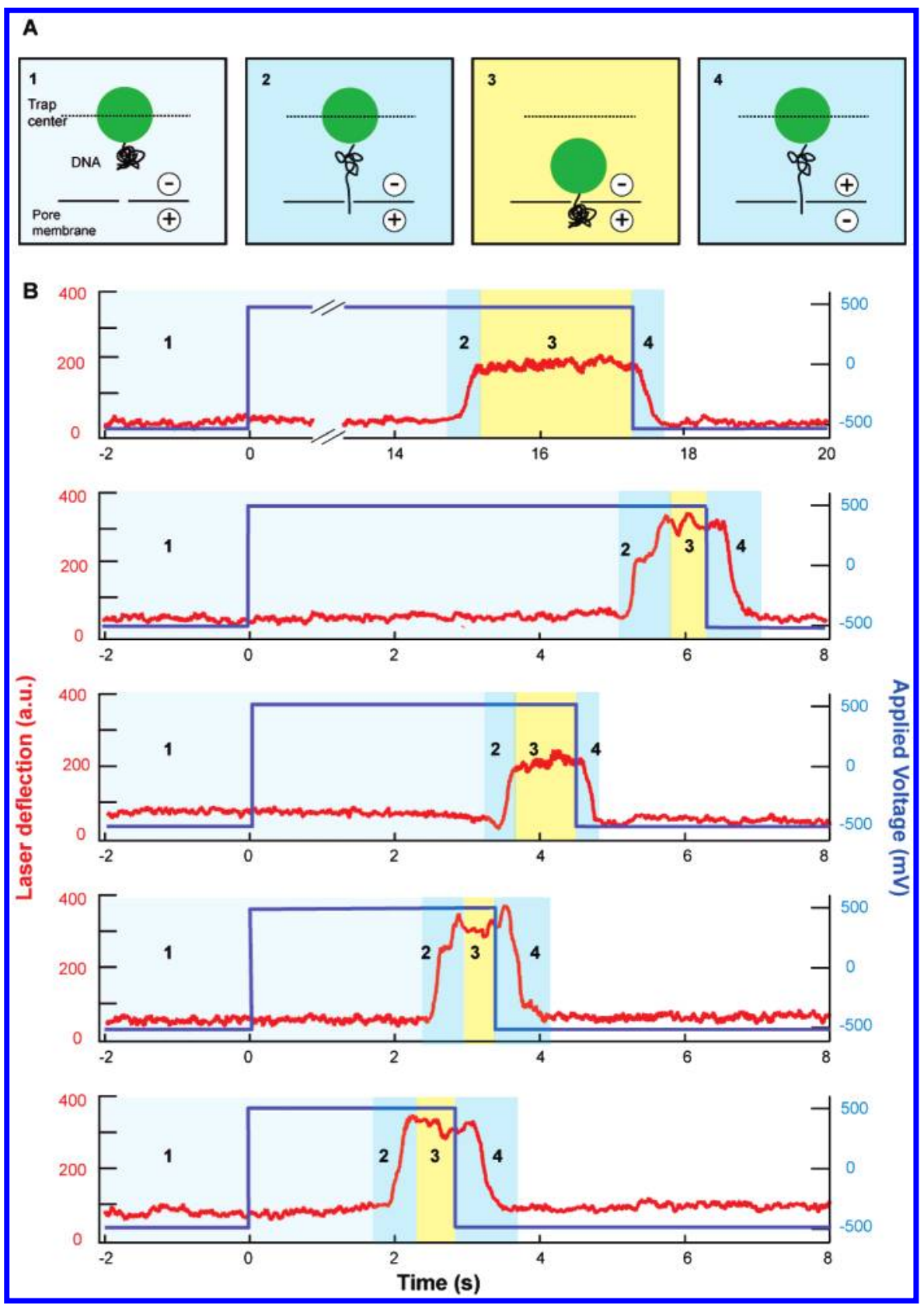

Figure 2. Traces of individual threading events. (A) Schematic of DNA threading experiment. The bead is held in the optical trap, a fixed distance from the nanopore (drawing 1). An external potential is applied across the nanopore, and the DNA is begins to thread (drawing 2). The DNA translocates through the pore, pulling the optically trapped bead along with it, until the bead reaches the nanopore membrane (drawing 3). Once the potential is reversed, the bead relaxes back into the center of the optical trap (drawing 4). Individual threading traces are shown in panel (B) The exiting optical trapping laser light (red trace) is monitored during the experiment. Motion of the bead in the trap modulates the laser deflection. At $t=0 \mathrm{~s}$, a positive potential of $500 \mathrm{mV}$ is applied. After a few seconds, the DNA is captured by the nanopore and translocates. The electric force overpowers the optical trap, pulling the bead toward the nanopore membrane. This motion of the bead out of the optical trap produces a large deflection in the trapping laser beam, which reverses when the bead returns to the trap center.

by 5,9 , and $30 \mathrm{~nm}$ nanopores as a function of initial distance. The distance between the pore and the pore-proximal side of the bead is reported in units of DNA radius of gyration $\left(R_{\mathrm{g}}\right)$. Our full-length $\lambda$-DNA $(L=16.4 \mu \mathrm{m})$ and restrictiondigested $\lambda$-DNA $(L=11 \mu \mathrm{m})$ have $R_{\mathrm{g}}=0.85$ and $0.67 \mu \mathrm{m}$, respectively. First, we trapped a bead and brought it close to the pore, turned on the voltage, and checked for threading. If we saw threading, then we used that same bead over and over again, as follows. We positioned the bead at some initial distance from the pore, turned on the voltage, and measured the time it took for threading to commence. This procedure allowed us to construct histograms of capture probability versus initial distance (Figure 3); each histogram was constructed with data from about five beads per pore diameter and at least 10 capture events per bead.

Capture and subsequent threading was observed for all beads and all pore diameters when the beads were placed 1 $R_{\mathrm{g}}$ from the pore. Capture efficiency dropped with increasing 


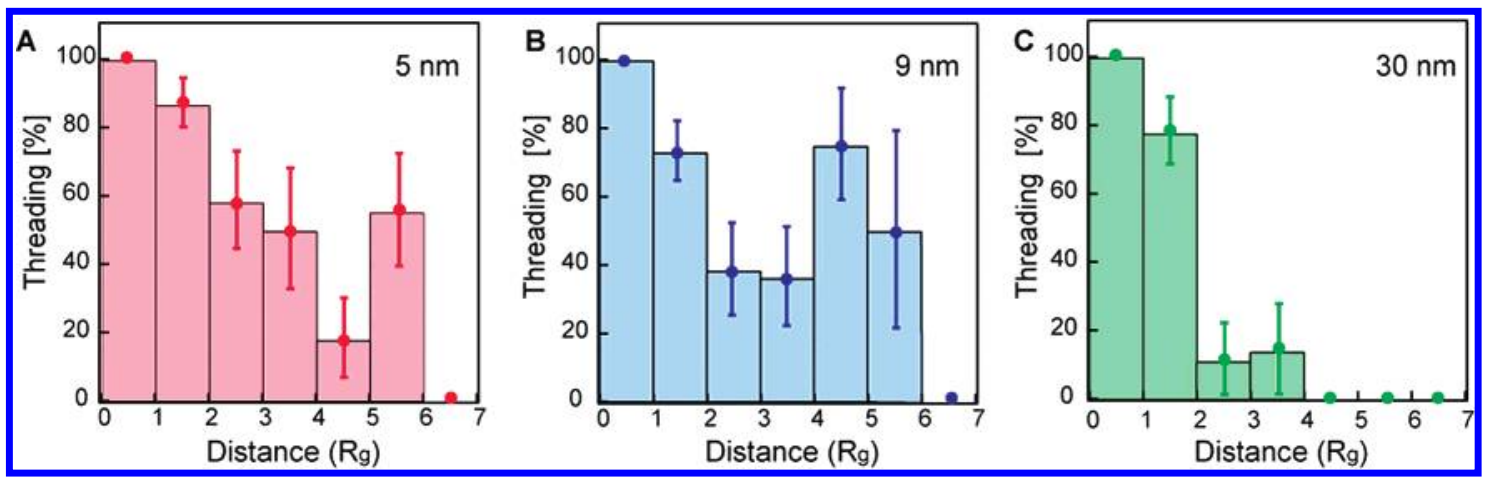

Figure 3. Capture rates by pore size. Bar charts showing percentage and standard deviation of DNA molecules which thread within $30 \mathrm{~s}$ at distances from 1 to 7 times the radius of gyration of the molecule. (A) Capture in $5 \mathrm{~nm}$ diameter pores. (B) Capture for $9 \mathrm{~nm}$ pores. (C) Capture in $30 \mathrm{~nm}$ pores. Applied voltage is $500 \mathrm{mV}$ for the $5 \mathrm{~nm}$ pore experiments and $300 \mathrm{mV}$ for larger pores. Threading percentages reach $100 \%$ at distances of $1 R_{\mathrm{g}}$ or less and generally decreases at larger distances.

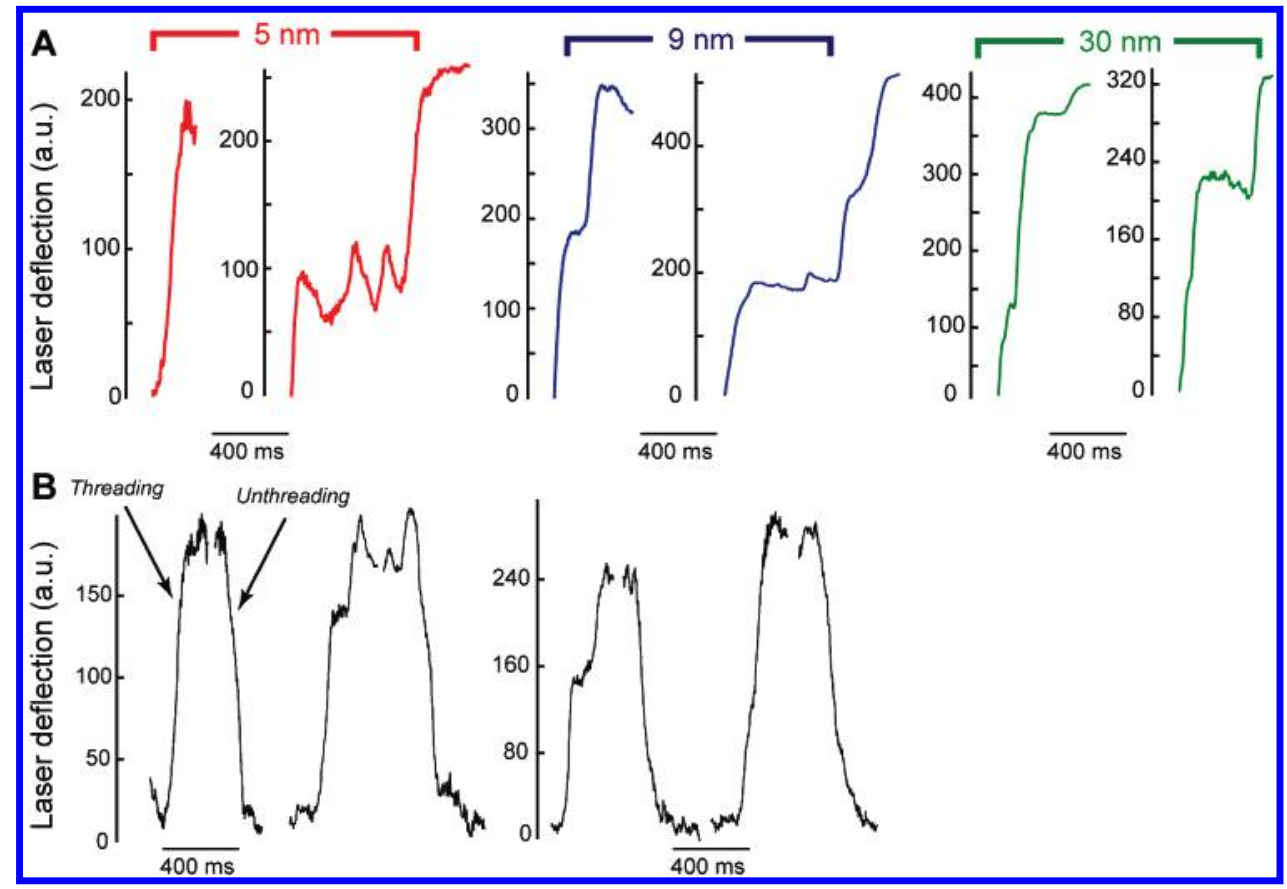

Figure 4. Individual translocation traces as monitored by changes in the laser deflection signal. As DNA translocates the pore, the attached bead moves out of the center of the optical trap, deflecting the trapping laser beam. Changes in the deflection signal indicate progression of the polymer through the pore, and plateaus indicate pauses in translocation. (A) Traces showing translocation of $11 \mu \mathrm{m}$ DNA through a $5 \mathrm{~nm}$ pore (red), a $9 \mathrm{~nm}$ pore (blue), and a $30 \mathrm{~nm}$ pore (green). (B) Traces of threading and retraction of individual DNA molecules through $5 \mathrm{~nm}$ pores.

distance, but more slowly than expected. In our geometry, the majority of the voltage drop is across the nanopore, and the electric field decays quickly $\left(\sim d_{\mathrm{p}}{ }^{2} / d^{2}\right)$ away from the mouth of the pore, where $d_{\mathrm{p}}$ is the pore diameter and $d$ the distance from the pore. ${ }^{16}$ Moreover, the DNA molecule in solution should be rather compact, and large thermal fluctuations greatly extending its length are unlikely. Nevertheless, we observed capture within $30 \mathrm{~s}$ of DNA molecules placed up to $6 R_{\mathrm{g}}$ from the nanopore. At these distances, the electric field decays to roughly $10^{-4} \%$ of its value at the nanopore. The time scales expected for a thermal fluctuation to produce a $6 R_{\mathrm{g}}$ excursion are on order of hours, as estimated by dividing the Zimm relaxation time ${ }^{17}$ by the probability of a $6 R_{\mathrm{g}}$ excursion as given by the freely-jointed-chain model. Monte Carlo simulations of a thermally fluctuating freelyjointed-chain confirm this estimate (Supporting Information).
We surmised that the high capture rates of DNA anchored far from the pore were influenced by fluid flows in the chamber. Such large DNA capture regions $(r \sim 3 \mu \mathrm{m})$ have been observed previously ${ }^{18}$ in a time-resolved fluorescence study of freely diffusing $\lambda$-DNA and alumina coated nanopores. These observations are supported by recent theoretical calculations ${ }^{19}$ showing that DNA translocation through pores carrying a positive surface charge is accompanied by an electro-osmotic flow (EOF) in the same direction as the translocation. The velocity gradient of this EOF around the nanopore may create an adsorbing region with a radius comparable in size to the $R_{\mathrm{g}}$ of the molecule.

In addition to the successful capture of DNA initially far from the pore, the experiments revealed another feature of the capture process. Especially for the small pores, capture required significantly higher potentials than threading. A 


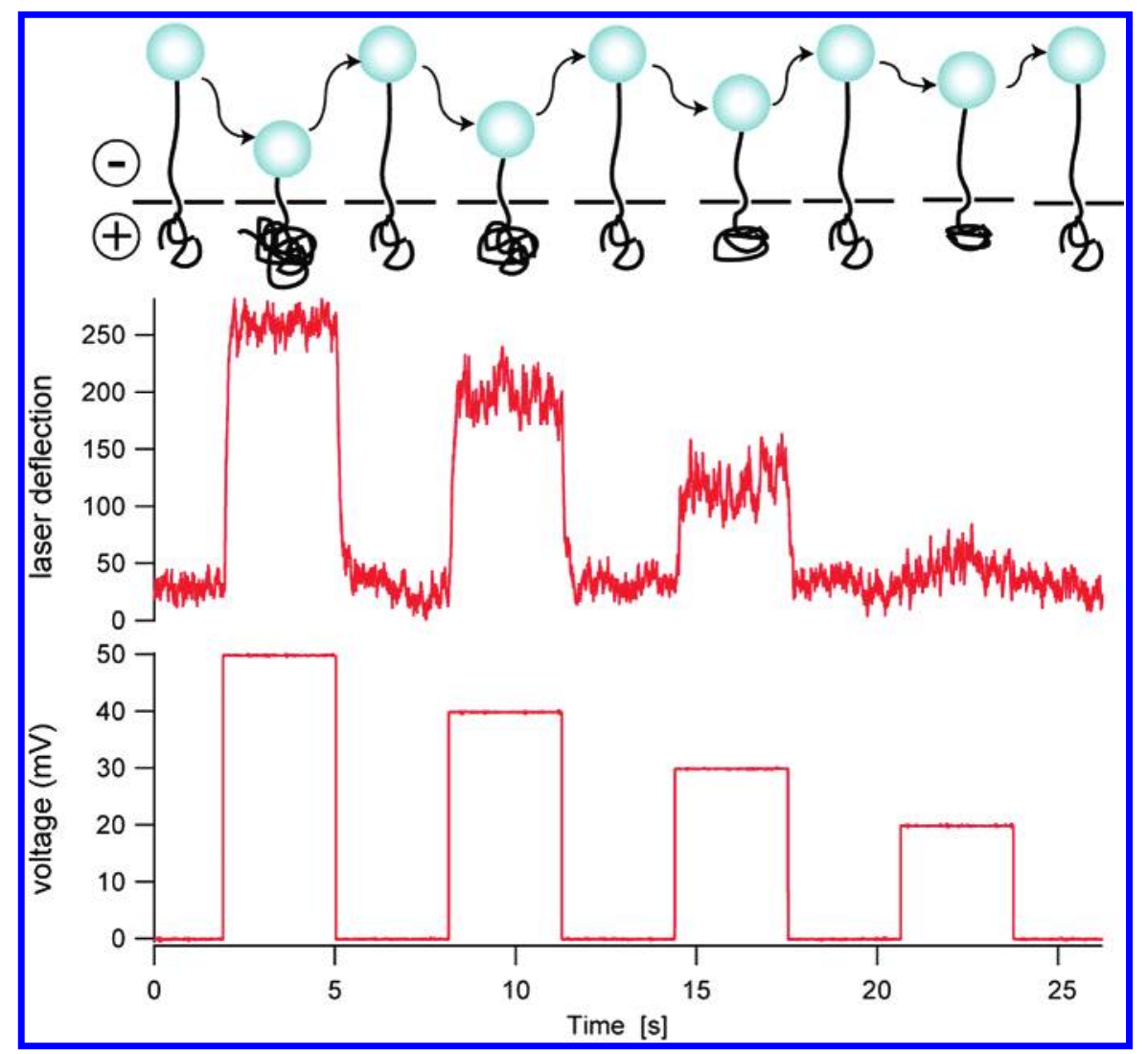

Figure 5. DNA "flossing". DNA attached to a trapped bead is held near the nanopore. Once the DNA is threaded, it can be held in the pore at low applied voltage. Small increases in voltage cause small changes in displacement of the bead with respect to the trap center, as seen by the changes of the laser deflection (in our setup, increases of the beam deflection correspond to movement of the bead toward the trapping laser and also toward the membrane).

threaded DNA molecule could be held in a nanopore with a few tens of $\mathrm{mV}$, but potentials of $0.3 \mathrm{~V}$ were needed for efficient capture (defined as $100 \%$ capture within $1 R_{\mathrm{g}}$ ) in 9 and $30 \mathrm{~nm}$ pores, and $0.5 \mathrm{~V}$ were required for efficient capture in $5 \mathrm{~nm}$ pores. A single bead placed at distances greater than $1 R_{\mathrm{g}}$ from our smallest pores exhibited significantly increased capture efficiency under $0.5 \mathrm{~V}$ (6 out of 8 attempts successful with $0.5 \mathrm{~V}$; 1 out of 7 attempts successful with $0.3 \mathrm{~V})$. An intriguing explanation for this increase in threshold potential is that capture of DNA in a bent configuration is energetically costly. Translocation of dsDNA through $10 \mathrm{~nm}$ pores in a bent conformation was first observed by $\mathrm{Li}$ et al. ${ }^{6}$; more recently, studies on dsDNA translocation through synthetic nanopores at $0.3 \mathrm{~V}$ show that translocation in a bent conformation is possible through $6.5 \mathrm{~nm}$ pores, but not through $4 \mathrm{~nm}$ pores. ${ }^{20}$

The data support the following general picture of DNA capture in our system: even far from the pore (e.g., 4 micrometers), the DNA feels forces that bias it to the pore. Once close to the pore, the DNA can enter the pore endfirst or anywhere along its length, by bending. The smallest pores have increased capture threshold voltage, perhaps due to the energetic cost of sharply bending DNA.

Turning to the translocation process, we set out to determine the DNA's translocation speed through the pore. Using the known length $(11 \mu \mathrm{m})$ of the DNA polymer along with the average time for each translocation event (less the average total pause length per event, see below), we obtained estimates for the DNA translocation speeds: $50 \mu \mathrm{m} / \mathrm{s}$, or approximately $150 \mathrm{bp} / \mathrm{ms}(11 \mu \mathrm{m}$ DNA, $5 \mathrm{~nm}$ pore, $N=$ 36). The translocation speeds of DNA through nanopores in our geometry are thus $\sim 200$ times slower than translocation speeds typical of free DNA. Because the DNA can be captured sideways as well as at the end, this value is a conservative measure of translocation speed and likely overestimates the true translocation speed, which is good from a device perspective. In principle, the decrease in translocation time converts directly into an increase in sequencing accuracy: a $10 \mathrm{MHz}$ electronic sampling rate provides statistics of $N=3$ measurements per base of a free molecule and about 200 times as many for a molecule attached to a trapped bead.

Not only was it possible to slow polymer propagation through the pore, but it was also possible to repeatedly characterize the same piece of DNA. In these "flossing" experiments (Figure 5), we brought the DNA-coated bead into proximity of the pore, turned on the voltage, and waited, as in Figure 2. The polymer threaded after the typical wait times of several seconds to tens of seconds (as in Figure 3). Then, we turned off the external potential and let the bead relax into the trap center. So far, the experiment was done precisely as described in Figure 2. Now, unlike in Figure 2, we turned the voltage back on (Figure 5, $t=2 \mathrm{~s}$ ), and the bead was instantaneously pulled toward the pore. 
The rapid response of the bead to the external potential jump suggests that the DNA never completely left the pore and immediately began to translocate once the external potential was turned back on. A second observation supports this interpretation. Unlike the polymer capture experiments (e.g., Figure 2), which involve voltages of hundreds of $\mathrm{mV}$, these repeated cycles of threading and retraction require only tens of $\mathrm{mV}$, presumably because the DNA is already in the pore. This DNA "flossing" could be repeated numerous times; different values of the external potential led to different laser beam deflections (and thus forces on the bead), but the instantaneous response of the bead remained. The instantaneous bead response was destroyed by moving the trapped bead far from the pore between voltage jumps, presumably because this completely freed the DNA, which must then be recaptured by the pore. Repeated cycles of threading and retraction ("flossing"), as shown in Figure 5, should increase the signal-to-noise of simultaneous current measurements in direct proportion to the number of times the process is repeated.

There are disadvantages to combining nanopores with optical tweezers, including increased instrument complexity and effects of the laser on noise levels. Laser light has been identified as a source of low frequency noise and variation in ionic current through silicon nitride nanopores. ${ }^{21}$ Another challenge introduced in our geometry is the pausing during DNA translocation. Figure 4 shows several individual traces of this translocation process. As can be seen in this series of traces, DNA does not always translocate smoothly, and many translocations are interrupted by pauses of varying length. We found that many pauses ( $\sim 40 \%$ ) lasted less than $50 \mathrm{~ms}$, a majority (68\%) last less than $100 \mathrm{~ms}$, and less than 5\% last $400 \mathrm{~ms}$ or longer. Pausing can occur multiple times during translocation events and can dominate the total event time. The average translocation time, neglecting these pauses, is $220 \mathrm{~ms}$ (std $170 \mathrm{~ms}$ ); the average total translocation time is $410 \mathrm{~ms}$ ( $\mathrm{std} 380 \mathrm{~ms}$ ).

Possible causes of kinetic barriers leading to the pauses are sticking of the DNA to the nanopore surface, the presence of entropic barriers resulting from restriction of the number of DNA conformations near and inside the nanopore, or pseudoknots in the DNA. Sticking of the DNA to the surface of the pore has been observed in molecular dynamics simulations of short segments of DNA translocating through synthetic pores. ${ }^{22} \mathrm{We}$ favor sticking as the explanation of the pauses because they can be very long-lived and are spatially well-localized and are not unexpected due to the positive surface charge of the pore. We could sometimes unstick the DNA mechanically, either by pulling sideways on the bead with the optical trap, increasing laser power, or both. Going forward with this technology, it will be important to find ways of reducing bead-to-membrane sticking and DNA to membrane interactions.

Now, translocation speeds of DNA through nanopores can be controlled and a single DNA molecule can be repeatedly flossed back and forth though the pore, removing one of several barriers to DNA sequencing using nanopores. This work points to the need for improved pore surface chemistries to reduce nonspecific adhesion; by reducing the net translocation force on the polymer, kinetic barriers become longerlived, assuming a standard exponential dependence of the adhesion time on the force. ${ }^{23}$ Future nanopore DNA and protein sequencing geometries may well exploit optical trapping for rate control and sample presentation, but they will also need to feature better passivated surfaces to ensure smooth polymer progression.

Acknowledgment. We thank the reviewer who suggested the Zimm relaxation time argument to estimate polymer fluctuation times and for extensive comments and suggestions. This work could not have been done without the generous support of Prof. Daniel Branton (Harvard University), who provided several nanopores and also assisted with subsequent pore fabrication and the construction of our ALD system. We thank the National Center for Electron Microscopy (NCEM) at LBL, and the Electron Microscopy Lab (EML) at UC Berkeley for use of their facilities. We thank Agilent Technologies for fabrication of nanopore chips. For financial support, we thank the UC Biotechnology Research and Education Program (E.H.T.) the Swiss National Science Foundation (A.R.), and the NSF graduate fellowship (D.S.). This work was supported in part by the University of California, Berkeley, the Hellman Faculty Fund, the Sloan and Searle foundations (JTL), and the DOE Office of Science, Energy Biosciences Program KC0304, DE-AC0376 SF00098.

Supporting Information Available: Fabrication of nanopores, chamber design, nanopore properties, instrumentation, Monte Carlo simulations. This material is available free of charge via the Internet at http://pubs.acs.org.

\section{References}

(1) Li, J. L.; Stein, D.; Qun, C.; Brandin, E.; Huang, A.; Wang, H.; Branton, D.; Golovchenko, J. Biophys. J. 2003, 84, 134A-135A.

(2) Fologea, D.; Gershow, M.; Ledden, B.; McNabb, D. S.; Golovchenko, J. A.; Li, J. L. Nano Lett. 2005, 5, 1905-1909.

(3) Stein, D.; van der Heyden, F. H. J.; Koopmans, W. J. A.; Dekker, C. Proc. Natl. Acad. Sci. U.S.A. 2006, 103, 15853-15858.

(4) Fan, R.; Karnik, R.; Yue, M.; Li, D. Y.; Majumdar, A.; Yang, P. D. Nano Lett. 2005, 5, 1633-1637.

(5) Karnik, R.; Fan, R.; Yue, M.; Li, D.; Yang, P.; Majumdar, A. Nano Lett. 2005, 5, 943-948.

(6) Li, J. L.; Gershow, M.; Stein, D.; Brandin, E.; Golovchenko, J. A Nat. Mater. 2003, 2, 611-615.

(7) Storm, A. J.; Storm, C.; Chen, J. H.; Zandbergen, H.; Joanny, J. F.; Dekker, C. Nano Lett. 2005, 5, 1193-1197.

(8) Li, J.; Stein, D.; McMullan, C.; Branton, D.; Aziz, M. J.; Golovchenko, J. A. Nature 2001, 412, 166-169.

(9) Branton, D.; Golovchenko, J. Nature 1999, 398, 660-661.

(10) Meller, A.; Nivon, L.; Branton, D. Phvs. Rev. Lett. 2001, 86, 34353438

(11) Fologea, D.; Uplinger, J.; Thomas, B.; McNabb, D. S.; Li, J. L. Nano Lett. 2005, 5, 1734-1737.

(12) Keyser, U. F.; Koeleman, B. N.; Van Dorp, S.; Krapf, D.; Smeets, R. M.; Lemay, S. G.; Dekker, N. H. and Dekker, C. Nat.Phvs. 2006, 2, 473-477.

(13) Neuman, K. C.; Block, S. M. Rev. Sci. Instrum. 2004, 75, 27872809.

(14) Pauzauskie, P. J.; Radenovic, A.; Trepagnier, E.; Shroff, H.; Yang, P.; Liphardt, J. Nat. Mater. 2006, 5, 97-101.

(15) Chen, P.; Mitsui, T.; Farmer, D. B.; Golovchenko, J.; Gordon, R. G.; Branton, D. Nano Lett. 2004, 4, 1333-1337.

(16) Lee, S.; Zhang, Y. H.; White, H. S.; Harrell, C. C.; Martin, C. R. Anal. Chem. 2004, 76, 6108-6115. 
(17) Do, M.; Edwards, S. The Theory of Polymer Dynamics; Clarendon: Oxford, 1986.

(18) Chen, C. M. Physica A 2005, 350, 95-107.

(19) Wong, C. T. A.; Muthukumara, M. J. Chem. Phys. 2007, 126, 164903-1-164903-6.

(20) Wanunu, M.; Meller, A. Biophysical Society 51st Annual Meeting, Baltimore, MD, March 3-7, 2007.
(21) Smeets, R. M. M.; Keyser, U. F.; Wu, M. Y.; Dekker, N. H.; Dekker, C. Phys. Rev. Lett. 2006, 97, 088101.

(22) Aksimentiev, A.; Heng, J. B.; Timp, G.; Schulten, K. Biophys. J 2004, 87, 2086-2097.

(23) Bell, G. I. Science 1978, 200, 61-627.

NL0714334 